\title{
Síntomas depresivos, riesgo de abandono y mala adherencia al tratamiento en pacientes con tuberculosis sensible en un centro de salud de Lima, 2016-2020
}

Depressive symptoms, risk of abandonment and poor adherence to treatment in patients with sensitive tuberculosis in a health center in Lima, 2016-2020

Mauro Jonis-Jiménez ${ }^{1, a}$, Roxana S. Guzman-Reinoso ${ }^{1, a}$, Félix K. Llanos-Tejada ${ }^{2,3, b}$

RESUMEN

Objetivo: Determinar si la presencia de síntomas depresivos es un factor asociado al abandono y a la pobre adherencia al tratamiento en pacientes con TB sensible en un centro de salud de Lima entre 2016-2020. Materiales $\boldsymbol{y}$ métodos: Estudio de tipo no experimental, analítico, de cohorte, retrospectivo. En el Centro de Salud MaternoInfantil Buenos Aires de Villa. La información fue recogida de las historias clínicas, se estimó el riesgo para cohorte (Riesgo Relativo) y niveles de significancia estadística, con ayuda del programa SPSS Statistics 25. Resultados: La frecuencia de abandono del tratamiento fue de $18,3 \%(n=28)$, de pobre adherencia $42,5 \%(n=65)$ y de depresión $53,6 \%(n=82)$. Los síntomas depresivos se asociaron a abandono al tratamiento $[\mathrm{RR}=2,16 ;$ IC95\% $(1,01-4,60)]$ y a pobre adherencia al tratamiento $[\mathrm{RR}=2,09 ; \mathrm{IC} 95 \%(1,36-3,22)]$. Conclusiones: La depresión es un factor de riesgo significativamente asociado a abandono y a pobre adherencia al tratamiento en pacientes con tuberculosis sensible.

PALABRAS CLAVE: Tuberculosis, depresión, abandono, adherencia, tratamiento.

\section{SUMMARY}

Objective: To determine if the presence of depressive symptoms is a factor associated with abandonment or poor adherence to treatment in sensitive TB patients in a Lima health center, between 2016-2020. Materials and methods: A non-experimental, analytical, cohort, retrospective study carried out. At the Centro de Salud MaternoInfantil Buenos Aires de Villa. The information was collected from the medical records, a cohort risk estimate (Relative Risk) and levels of statistical significance were performed, with the SPSS Statistics 25 program. Results: The frequency of treatment abandonment was $18.3 \%(\mathrm{n}=28)$, poor adherence $42.5 \%(\mathrm{n}=65)$ and depression $53.6 \%$ $(\mathrm{n}=82)$. Depressive symptoms were associated with treatment abandonment $[\mathrm{RR}=2.16 ; 95 \% \mathrm{CI}(1.01-4.60)]$ and poor treatment adherence $[\mathrm{RR}=2.09 ; 95 \% \mathrm{CI}(1.36-3.22)]$. Conclusions: Depression is a risk factor significantly associated with abandonment and poor adherence to treatment in patients with sensitive tuberculosis.

KEYWORDS: Tuberculosis, depression, abandonment, adherence, treatment.

Facultad de Medicina Humana, Universidad Ricardo Palma. Lima, Perú.

2 Hospital Nacional Dos de Mayo. Lima, Perú.

3 Instituto de Investigación de Ciencias Biomédicas, Facultad de Medicina Humana, Universidad Ricardo Palma. Lima, Perú.

a Médico Cirujano; ${ }^{\text {b }}$ Médico Neumólogo. 


\section{INTRODUCCIÓN}

La depresión es un grave problema de salud pública que va en aumento, se estima que afecta a más de 300 millones de personas en el mundo y es una causa de discapacidad, además de contribuir a la carga mundial general de morbilidad (1). La depresión afecta las áreas cognitiva, afectiva, motivacional y somática lo que compromete la salud y las medidas para mejorarla (2). Los pacientes con depresión muestran menor adherencia y mayor abandono de los tratamientos que les son indicados. Estas conductas aumentan en las patologías crónicas, cuyo tratamiento es de mayor duración y el éxito de este depende de su término y estricto cumplimiento (3).

Un caso de patología crónica, con éxito terapéutico alto, que depende de la adherencia y del término del tratamiento, es la tuberculosis (TB). La TB es una enfermedad infectocontagiosa prevenible y curable que afecta en mayor frecuencia a los países en vías de desarrollo. La Organización Mundial de la Salud (OMS) reporta que el año 2018 alrededor de 10 millones de personas se enfermaron de TB y 1,2 millones fallecieron, siendo la principal causa de muerte infecciosa del mundo $(4,5)$. En Perú, el año 2019 se reportaron 42940 casos de TB, ocupando el décimo primer lugar de las causas de muerte en general $(4,5)$.

Así, si hay abandono de tratamiento o se recibe irregularmente, se desarrollan formas de resistencia a fármacos, que requieren un tratamiento de mayor duración, mayor complejidad y menor tasa de curación (4). Lamentablemente, el aumento de los casos de las formas resistentes de TB se debe principalmente al abandono y al tratamiento irregular de las formas sensibles (5). El abandono y la mala adherencia son de causa multifactorial, incluyendo alteraciones de la salud mental (6). La principal causa de alteración de la salud mental es la depresión y su frecuencia es alta en la población con tuberculosis (7). Otros factores, evaluados en estudios previos $(8,9)$, son el gasto de viaje para recibir el tratamiento, género masculino, bajos niveles de conocimiento acerca de la enfermedad, dificultad en la comunicación con los pacientes, alcoholismo y abandono social, considerado como los principales determinantes sociales de no adherencia del tratamiento.

El objetivo de esta investigación fue determinar si la presencia de síntomas depresivos es un factor asociado al abandono y a la mala adherencia del tratamiento antituberculoso sensible.

\section{MATERIAL Y MÉTODOS}

El diseño de la presente investigación es de tipo analítico de cohorte retrospectivo. La muestra estuvo conformada por los pacientes que se atendieron en la Estrategia de Tuberculosis del Centro de Salud Materno Infantil Buenos Aires de Villa, en el periodo 2016-2020. Se realizó un muestreo no probabilístico por conveniencia tipo censo. Se consideró como criterios de inclusión a los pacientes ingresados como caso nuevo, que reciben el tratamiento para tuberculosis sensible, de cualquier localización y que cuente con evaluación por psicología en las primeras 2 semanas desde el inicio del tratamiento. La información que se recogió de las historias clínicas fue el resultado de la evaluación psicológica, mediante el test de SQR-18, para determinar depresión. Otras variables fueron edad, sexo, diabetes mellitus, VIH, localización de la enfermedad y, del registro de la Tarjeta de Tratamiento, se revisó la asistencia a las tomas de la medicación, definiendo el abandono, de acuerdo a la normatividad vigente, como dejar de recibir tratamiento al menos 30 días consecutivos, y la adherencia como presentar 3 faltas en la fase intensiva y/o 5 faltas durante el tratamiento. Estos datos fueron ingresados en el programa estadístico SPSS, donde se determinaron la frecuencia y para la estimación de riesgo entre las variables se aplicó la medida de asociación Riesgo Relativo (RR) con su respectivo intervalo de confianza. Se consideró estadísticamente significativo un valor $\mathrm{p}$ de 0,05 . Las variables que mostraron significancia en el análisis bivariado se introdujeron en un análisis multivariado, mediante regresión logística. El proyecto de investigación fue revisado y aprobado por un Comité de Ética.

\section{RESULTADOS}

La población estudiada fue de 153 pacientes, los que cumplían con los criterios de inclusión. Se midió la frecuencia de depresión, abandono del tratamiento, mala adherencia al tratamiento, coexistencia de Diabetes Mellitus, coinfección con VIH, sexo, etapa de vida según edad, localización de la enfermedad y número de faltas a la toma del tratamiento (tabla 1). La media de la edad fue 36,94+/- 17.9 años, con un rango entre 14 y 87 años. Por otro lado, la media de faltas fue de 5,97 faltas durante el tratamiento. 
Tabla 1. Frecuencias de depresión, abandono del tratamiento, mala adherencia al tratamiento, número de faltas a la toma del tratamiento, edad según etapa de vida, sexo, diabetes mellitus, VIH y localización de la enfermedad.

\begin{tabular}{|c|c|c|c|}
\hline & & Frecuencia & Porcentaje \\
\hline & $\mathrm{Si}$ & 82 & $53,6 \%$ \\
\hline Depresión & No & 71 & $46,4 \%$ \\
\hline Abandono del & $\mathrm{Si}$ & 28 & $18,3 \%$ \\
\hline tratamiento & No & 125 & $81,7 \%$ \\
\hline \multirow{3}{*}{$\begin{array}{c}\text { Mala adherencia al } \\
\text { tratamiento }\end{array}$} & $\mathrm{Si}$ & 65 & $42,5 \%$ \\
\hline & No & 88 & $57,5 \%$ \\
\hline & Sin faltas & 65 & $42,5 \%$ \\
\hline \multirow{5}{*}{$\begin{array}{c}\text { Número de faltas a } \\
\text { la toma de } \\
\text { tratamiento }\end{array}$} & Hasta 10 faltas & 51 & $33,3 \%$ \\
\hline & De 10 a 20 faltas & 21 & $13,7 \%$ \\
\hline & De 20 a 30 faltas & 16 & $10,5 \%$ \\
\hline & Adolescencia & 13 & $8,5 \%$ \\
\hline & Juventud & 43 & $28,1 \%$ \\
\hline \multirow{3}{*}{$\begin{array}{c}\text { Etapa de vida según } \\
\text { edad }\end{array}$} & Adultez & 74 & $48,4 \%$ \\
\hline & Adulto mayor & 23 & $15 \%$ \\
\hline & Masculino & 90 & $58,8 \%$ \\
\hline \multirow[t]{2}{*}{ Sexo } & Femenino & 63 & $41,2 \%$ \\
\hline & $\mathrm{Si}$ & 27 & $17,6 \%$ \\
\hline \multirow[t]{2}{*}{ Diabetes melitus } & No & 126 & $82,4 \%$ \\
\hline & $\mathrm{Si}$ & 8 & $5,2 \%$ \\
\hline VIH & No & 145 & $94,8 \%$ \\
\hline Localización de la & Pulmonar & 117 & $76,5 \%$ \\
\hline enfermedad & Extrapulmonar & 36 & $23,5 \%$ \\
\hline
\end{tabular}

Tabla 2. Análisis bivariado con respecto al abandono y mala adherencia al tratamiento.

\begin{tabular}{|c|c|c|c|c|c|}
\hline & \multicolumn{2}{|c|}{ Abandono del tratamiento } & \multicolumn{2}{|c|}{ Mala adherencia al tratamiento } \\
\hline & & $\mathbf{R R}$ & IC $95 \%$ & RR & IC $95 \%$ \\
\hline \multicolumn{2}{|c|}{ Depresión } & 2,165 & $1,017-4,609$ & 2,096 & $1,364-3,222$ \\
\hline \multicolumn{2}{|r|}{ Sexo } & 2,100 & $0,951-4,639$ & 1,196 & $0,812-1,762$ \\
\hline \multicolumn{2}{|c|}{ Edad menor de 36 años } & 1,226 & $0,608-2,476$ & 1,182 & $0,819-1,707$ \\
\hline \multicolumn{2}{|c|}{ Diabetes mellitus } & 1,867 & $0,921-3,784$ & 1,524 & $1,040-2,232$ \\
\hline \multicolumn{2}{|r|}{ VIH } & 1,394 & $0,400-4,865$ & 1,843 & $1,180-2,878$ \\
\hline \multicolumn{2}{|c|}{ Localización de la enfermedad } & 4,000 & $0,997-16,041$ & 0,869 & $0,578-1,306$ \\
\hline \multirow{6}{*}{$\begin{array}{l}\text { Número de } \\
\text { faltas a la } \\
\text { toma de } \\
\text { tratamient } \\
\text { o }\end{array}$} & Más de 1 falta & 19,943 & $2,781-143,009$ & & \\
\hline & Más de 2 faltas & 32,870 & $4,582-235,783$ & & \\
\hline & Más de 3 faltas & 36,554 & $5,098-262,121$ & & \\
\hline & Más de 4 faltas & 44,224 & $6,173-316,809$ & & \\
\hline & Más de 5 faltas & 48,109 & $6,720-344,431$ & & \\
\hline & Más de 6 faltas & 55,620 & $7,779-397,69$ & & \\
\hline \multicolumn{2}{|c|}{ Mala adherencia al tratamiento } & 36,554 & $5,098-262,121$ & & \\
\hline
\end{tabular}


Tabla 3. Análisis multivariado respecto a abandono y mala adherencia al tratamiento.

\begin{tabular}{ccccc}
\hline & $\begin{array}{c}\text { Abandono del Tratamiento } \\
\text { RR }\end{array}$ & $\begin{array}{c}\text { Mala adherencia al tratamiento } \\
\text { IC 95\% }\end{array}$ & RR & IC 95\% \\
\hline Depresión & 1,14 & $0,40-3,28$ & 3,41 & $1,69-6,86$ \\
Mala adherencia & 0,01 & $0,002-0,131$ & & \\
Diabetes Mellitus & & & 0,44 & $0,18-1,09$ \\
VIH & & & 0,20 & $0,037-1,15$ \\
\hline
\end{tabular}

El análisis bivariado respecto al abandono del tratamiento nos reportó que presentar depresión (RR: 2.16, $\mathrm{p}<0.05$ ), mala adherencia (RR: 36.5, $\mathrm{p}<0.05$ ) y al menos una falta a la toma del tratamiento (RR: $19.9, \mathrm{p}<0.05)$ fueron factores asociados. Respecto a presentar mala adherencia al tratamiento se encontró que presentar depresión (RR: 2.1, $\mathrm{p}<0.05$ ), Diabetes Mellitus (RR: 1.5, p<0.05), coinfección con VIH (RR: $1.8, \mathrm{p}<0.05$ ) fueron factores asociados (tabla 2). Las variables edad, sexo y localización de enfermedad no obtuvieron significancia estadística.

La tabla 3 muestra el análisis multivariado, que encontró que la depresión es un factor de riesgo para la mala adherencia al tratamiento antituberculoso (OR: $3.4, \mathrm{p}<0.05)$.

\section{DISCUSIÓN}

El objetivo del presente fue determinar si la presencia de síntomas depresivos es un factor de riesgo para mala adherencia y abandono de tratamiento TB. $\mathrm{Y}$, aunque no pudimos determinar asociación con abandono, sí se determinó asociación entre síntomas depresivos y mala adherencia al tratamiento.

Hallamos que la frecuencia de depresión en los pacientes en tratamiento antituberculoso de primera línea fue un poco superior a la mitad $(53,6 \%)$. Otros estudios encuentran diversas frecuencias, como Duko y col., (43,4\%) (10), Aamir y col., (72\%) (11), Kehbila y col., (61,1\%) (12), Molla y col., (31,1\%) (13), Doherty y col., (70\%) (14). Esta variabilidad se puede explicar por los diversos instrumentos utilizados para el diagnóstico de síntomas depresivos o depresión en los diferentes estudios, como el cuestionario PHQ-9, cuestionario de Beck, escala de Hamilton para depresión, escala de depresión y ansiedad de Goldberg, instrumentos diseñados por las instituciones que dirigen la normativa de salud de los países donde se llevaron a cabo las investigaciones y el cuestionario de síntomas SRQ-18 que fue utilizado en el presente estudio. Un factor para tomar en cuenta es el momento del tratamiento en que se realizó el diagnóstico de depresión, ya que pueden variar las frecuencias; en el presente trabajo se seleccionaron a los pacientes que fueron evaluados por sicología a las dos semanas de inicio del tratamiento.

Nuestro estudio pudo determinar que la presencia de depresión es un factor de riesgo para el abandono y la mala adherencia al tratamiento antituberculoso de primera línea. Del mismo modo fue descrito, en un estudio realizado por Ambaw y col. (15), en el año 2018, cuyo objetivo era estudiar la influencia de la depresión en los pacientes con tuberculosis en su calidad de vida, concluyendo que los pacientes con depresión tienen hasta 9 veces más riesgo de abandonar el tratamiento. Respecto a la adherencia al tratamiento en la revisión sistemática realizada por Neves y col., (16), se concluyó que los pacientes con depresión tienen 2,6 veces más riesgo de no adherirse al tratamiento antituberculoso. Un estudio realizado por Llanos y col., (17), en un hospital de Lima mencionan "Pudimos determinar asociación estadísticamente significativa entre nivel de adherencia según MoriskyGreen y depresión según PHQ-9 $(\mathrm{p}<0,05)$ )". En la red de salud Almenara en el departamento de Lima, Morales (18) describió que los pacientes con depresión moderada tan solo presentan $17,65 \%$ de adherencia al tratamiento $(\mathrm{p}<0.05)$. Por último, otra revisión sistemática y metaanálisis, realizado por Ruiz y col. (19) concluyó que la depresión es un factor asociado a resultados negativos como mortalidad, abandono y fracaso del tratamiento, y respecto al abandono resultó ser un factor fuertemente asociado $\left[\mathrm{OR}=8,70 ; \mathrm{IC}_{95 \%}\right.$ $(6,50-11,64)]$. Nuestro análisis multivariado confirma que padecer de depresión es un factor de riesgo independiente a presentar mala adherencia durante el tratamiento de TB sensible.

La frecuencia de pacientes con mala adherencia al tratamiento fue de $42,5 \%$, y, el $96,4 \%$ de los pacientes que abandonaron presentaron mala adherencia durante el tratamiento, con hasta 36 veces más riesgo de abandonar el tratamiento. De manera similar Kehbila y 
Síntomas depresivos, riesgo de abandono y adherencia pobre al tratamiento en pacientes con tuberculosis sensible.

col., encontraron que llevar un tratamiento discontinuo es un factor de riesgo para el abandono del tratamiento (12). Por lo que consideramos importante mencionar que la frecuencia de $66 \%$ de mala adherencia al tratamiento en pacientes con TB MDR en el estudio de Maldonado (20), en el hospital Regional de Pucallpa, ya que el fracaso y el abandono del tratamiento es mayor en esta población y la tasa de curación es menor. Respecto a las faltas al tratamiento, menos de la mitad (42,5\% de los pacientes) no tuvo faltas a la toma del tratamiento, lo que podría definirse como adherencia adecuada al mismo. El análisis nos mostró que los pacientes con una o más faltas tienen hasta 19 veces más riesgo de abandonar el tratamiento y que este riesgo se incremente directamente proporcional al número de faltas. Cáceres y col., en su estudio describieron como resultado que presentar más de dos faltas al tratamiento es un factor asociado al abandono del tratamiento (23).

En cuanto a la variable sexo, el estudio arrojó que la mayoría de los pacientes que reciben tratamiento son de sexo masculino $(58,8 \%)$, sin poder encontrar riesgo estadísticamente significativo, pero sí incrementado, para abandono y mala adherencia al tratamiento. Faria y col. encontraron que el sexo masculino representa un factor asociado al abandono del tratamiento (21). Respecto a la adherencia al tratamiento Herrero y col. encontraron que el sexo masculino es un factor asociado a mala adherencia al tratamiento antituberculoso (22). Por otro lado, Kehbila y col., en un análisis multivariado mediante regresión, encontraron que el sexo femenino es un factor asociado al abandono del tratamiento, además de presentar 2,5 veces más riesgo de tener posibilidad de tener depresión (12). Es quizás por esta controversia, que nuestros hallazgos no han podido ser concluyentes respecto al sexo como factor de riesgo para abandono o mala adherencia.

La media de edad fue de 36,9 años, sin encontrar evidencia de riesgo ni para el abandono ni para la mala adherencia al tratamiento. Aunque, de acuerdo con lo esperado, se determinó que el $89 \%$ de los pacientes que abandonó el tratamiento son jóvenes y adultos (18-59 años). Un resultado similar al esperado por nosotros fue el hallado por Rivera y col. (24), en su estudio tener entre 28 y 37 años es un factor asociado con el abandono del tratamiento.

La localización, pulmonar o extrapulmonar, de la enfermedad no se asoció ni al abandono ni a la mala adherencia durante el tratamiento. El estudio de Soares y col. (25) determinó que los pacientes con ambas presentaciones tuvieron mayor frecuencia de abandono $(14,1 \%)$ que aquellos con presentación pulmonar $(11,2 \%)$ y extrapulmonar $(11,4 \%)$.

No pudimos determinar riesgo estadísticamente, aunque el $28,5 \%$ de los pacientes que abandonaron el tratamiento padecen de diabetes mellitus. Respecto a la mala adherencia, el $24,6 \%$ de los pacientes padece de diabetes mellitus, demostrando asociación y hasta 1,5 veces más riesgo. Maldonado (20), en su estudio en el hospital regional de la ciudad de Pucallpa, sobre la influencia de depresión en la adherencia al tratamiento en pacientes con TB MDR, encontró que la frecuencia de diabetes mellitus fue de $23 \%$. Rivera y col. (24), en Perú, buscaron determinar factores asociados al abandono del tratamiento de TB MDR, y encontraron que los pacientes con diabetes tienen hasta 6 veces más riesgo de abandonar el tratamiento. No hemos podido encontrar estudios respecto a riesgo de abandono al tratamiento sensible y padecer de diabetes mellitus.

La frecuencia de coinfección con VIH en los pacientes que abandonaron el tratamiento fue de 7,14\%, similar resultado fue descrito por Torres y col. (26) en su estudio en Chile sobre el perfil de los pacientes que abandonan el tratamiento, de los cuales $8,2 \%$ están infectado con VIH. En cuanto a presentar mala adherencia, los pacientes con VIH mostraron 1,8 veces más riesgo. En un país con alta carga de VIH como Camerún, Kehbila y col. (12), encontraron que los pacientes con VIH tienen 2,5 veces más riesgo de presentar mala adherencia al tratamiento. Una explicación a la falta de un resultado estadísticamente significativo sea por la poca muestra de pacientes con VIH en nuestra población, aunque no era el objetivo del presente estudio.

En conclusión, la presencia de síntomas depresivos es un factor de riesgo asociado al abandono y a la mala adherencia al tratamiento en pacientes TB sensible. $\mathrm{La}$ mala adherencia al tratamiento es un factor de riesgo asociado al abandono del tratamiento.

Una gran limitación del estudio es que solo se ha evaluado la población de un establecimiento de salud, lo que dificulta la extrapolación de nuestros resultados. Asimismo, no se ha podido determinar enfermedad reflejada en la salud mental, solo presencia o ausencia de síntomas depresivos.

El presente estudio forma parte de la tesis: GuzmánReinoso R. y Jonis-Jiménez M. "Depresión como factor asociado al abandono y a la mala adherencia del tratamiento en pacientes TB sensibles del Centro 
Materno Infantil Buenos Aires de Villa de Chorrillos, 2016-2020." [Tesis de Pregrado]. Lima: Facultad de Medicina Humana, Universidad Ricardo Palma; 2020. Declaración de financiamiento: Autofinanciamiento Conflicto de intereses: Los autores declaran NO tener ningún conflicto de interés respecto a la publicación del presente manuscrito.

\section{Correspondencia:}

Roxana S. Guzmán Reinoso

Dirección: Jr. Carlos Arrieta 367 Barranco, Lima-Perú Correo electrónico: rguzmanreinoso@gmail.com Teléfono: 51993004351

\section{REFERENCIAS BIBLIOGRÁFICAS}

1. Organización Mundial de la Salud. Depresión. Ginebra: Organización Mundial de la Salud; 2020. (Citado 30 de enero del 2020). Disponible en: https://www.who.int/es/news-room/fact-sheets/ detail/depression

2. Kaplan H. Compendio de Psiquiatría. Barcelona: Salvatierra; 2016.

3. Goldstein CM, Gathright EC, Gunstad J, et al. Depressive symptoms moderate the relationship between medication regimen complexity and objectively measured medication adherence in adults with heart failure. J Behav Med. 2017; 40: 602-611. doi: 10.1007/s10865-017-9829-z

4. Furin J, Cox H, Pai M. Tuberculosis. Lancet. 2019;393(10181):1642-1656. DOI: 10.1016/S01406736(19)30308-3

5. World Health Organization. Global tuberculosis report 2019. Ginebra: World Health Organization; 2019. (Citado 30 de enero del 2020). Disponible en: https://apps.who.int/iris/bitstream/handle/10665/ 329368/9789241565714-eng.pdf

6. Organización Mundial de la Salud. Adherencia a 1 os tratamientos a largo plazo: Pruebas para la acción. Ginebra: Organización Mundial de la Salud; 2004. (Citado 30 de enero del 2020). Disponible en: https:// www.paho.org/Spanish/AD/DPC/NC/nc-adherencia. pdf.

7. Peltzer K, Naidoo P, Matseke G, Louw J, Mchunu G, Tutshana B. Prevalence of psychological distress and associated factors in tuberculosis patients in public primary care clinics in South Africa. BMC Psychiatry. 2012;12(1):89. Doi: 10.1186/1471-244X12-86.

8. Alarcón V, Alarcón E, Figueroa C, Mendoza-Ticona A. Tuberculosis in Peru: epidemiological situation, progress and challenges for its control. Rev Peru Med Doi: 10.17843/rpmesp.2017.342.2384

9. Zevallos M. Factores asociados al Abandono del tratamiento Antituberculoso, esquema I, en la Red de salud San Juan de Lurigancho.Tesis para optar el grado de Maestro en Control de Enfermedades Infecciosas y Tropicales. Lima Perú: Universidad Peruana Cayetano Heredia; 2017. (Citado 30 de enero del 2020). Disponible en: http://repositorio.upch.edu.pe/ handle/upch/1030

10. Duko B, Gebeyehu A, Ayano G. Prevalence and correlates of depression and anxiety among patients with tuberculosis at Wolaita Sodo University Hospital and Sodo Health Center, WolaitaSodo, South Ethiopia, Cross sectional study. BMC Psychiatry. 2015; 15:214. Doi: 10.1186/s12888-015-0598-3.

11. Aamir S, Aisha. Co-morbid anxiety and depression among pulmonary tuberculosis patients. J Coll Physicians Surg Pak. 2010; 20(10):703-4. doi: 10.2010/JCPSP.703704

12. Kehbila J, Ekabe CJ, Aminde LN, Noubiap JJ, Fon PN, Monekosso GL. Prevalence and correlates of depressive symptoms in adult patients with pulmonary tuberculosis in the Southwest Region of Cameroon. Infect Dis Poverty. 2016; 5(1):51. Doi: 10.1186/s40249-016-0145-6.

13. Molla A, Mekuriaw B, Kerebih H. Depression and associated factors among patients with tuberculosis in Ethiopia: a cross-sectional study. Neuropsychiatric Disease and Treatment. 2019;15: 1887-1893. Doi: 10.2147/NDT.S208361

14. Doherty AM, Kelly J, McDonald C, O'Dywer AM, Keane J, Cooney J. A review of the interplay between tuberculosis and mental health. Gen Hosp Psychiatry. 2013;35(4):398-406. Doi: 10.1016/j. genhosppsych.2013.03.018

15. Ambaw F, Mayston R, Hanlon C, Medhin G, Alem A. Untreated depression and tuberculosis treatment outcomes, quality of life and disability, Ethiopia. Bull World Health Organ. 2018; 96(4):243. Doi:10.2471/BLT.17.206417

16. Neves LA, Reis RK, Gir E. Adesão ao tratamento por indivíduos com a co-infecção HIV/tuberculose: revisão integrativa da literatura. Rev Esc Enferm USP. 2010;44(4):1135-41. Doi: 10.1590/s008062342010000400041

17. Llanos-Tejada F, Ponce-Chang Cr. Depresión y adherencia en personas afectadas con tuberculosis: Una exploración preliminar de datos. Rev Neuropsiquiatr. 2019; 82(2): 104-109. Doi: 10.20453/ rnp.v82i2.3536.

18. Morales H. Influencia de la depresión sobre la adherencia al tratamiento en pacientes TB-MDR de la Red Almenara. Revista de la Facultad de Medicina Humana de la Universidad Ricardo Palma. 2015;1: 16-22.

19. Ruiz P, Cachay R, De la Flor A, Schwalb A, Ugarte C. Association between tuberculosis and depression on negative outcomes of tuberculosis treatment: A systematic review and meta-analysis. PLoS 
Síntomas depresivos, riesgo de abandono y adherencia pobre al tratamiento en pacientes con tuberculosis sensible.

One. 2020;15(1): e0227472. Doi: 10.1371/journal. pone. 0227472

20. Maldonado A, Lozano L. Influencia de la depresión sobre la adherencia al tratamiento en pacientes con tuberculosis multidrogoresistente en el Hospital Regional de Pucallpa en el periodo Enero 2014Diciembre 2015. Tesis Pregrado. Pucallpa: Universidad Nacional de Ucayali; 2016. (Citado 30 de enero del 2020). Disponible en: http://repositorio. unu.edu.pe/bitstream/handle/UNU/1611/ 000002027T.pdf? sequence $=1$ \&isAllowed $=\mathrm{y}$.

21. Faria N, Mota M, Marins R, Barbosa A, Soares L, Oliveira A, Souto J. Differences between risk factors associated with tuberculosis treatment abandonment and mortality. Pulm Med. 2015; 2015:546106. Doi: $10.1155 / 2015 / 546106$

22. Herrero M, Arrossi S, Ramos S, Braga J. Social determinants of nonadherence to tuberculosis treatment in Buenos Aires, Argentina. Cad Saúde Pública. 2015; 31(9): 1983-1994. (Citado 15 de diciembre del 2020). Disponible en: http://www. scielo.br/scielo.php?script=sci_arttext\&pid=S0102311X2015000901983\&lng=en.
23. Cáceres F, Orozco L. Incidencia y factores asociados al abandono del tratamiento antituberculoso. Biomédica. 2007; 27(4): 498-504. (Citado 15 de diciembre del 2020). Disponible en: http://www. scielo.org.co/scielo.php?script=sci_arttext\&pid= S0120-41572007000400005\&lng=en

24. Rivera O, Benites S, Mendigure J, Bonilla C.Abandono del tratamiento en tuberculosis multirresistente: factores asociados en una región con alta carga de la enfermedad en Perú. Biomédica. 2019; 39(2): 4457. Doi: 10.7705/biomedica.v39i3.4564.

25. Soares M, Amaral N, Zacarias A, Ribeiro L. Sociodemographic, clinical and epidemiological aspects of Tuberculosis treatment abandonment in Pernambuco, Brazil, 2001-2014. Epidemiol Serv Saude. 2017; 26(2):369-378. Doi: 10.5123/S167949742017000200014.

26. Torres Z, Herrera T. Perfil del paciente con tuberculosis que abandona el tratamiento en Chile: profile of patients in Chile. Rev chil enferm respir. 2015; 31(1): 52-57. (Citado 15 de diciembre del 2020). Disponible en: https://scielo. conicyt.cl/scielo.php?script=sci_arttext\&pid=S0717$73482015000100008 \& \operatorname{lng}=$ es.

Recibido: 02/02/2021

Aceptado: 23/12/2021 